\title{
PHOTOABSORPTION AND COMPTON SCATTERING IN IONIZATION OF HELIUM AT IIIGH PIIOTON ENERGIES
}

\author{
I.R. Anderason and J. Durgdörfor \\ Department of Physics and Astronomy, 401 A.H. Nieloen Bldg., \\ Univorulty of Tennessoe, Knuxvillo, TN 37000-1200, USA \\ and Oak Rdge National Laboratory, Oak Ridge, TN 37931-6377, USA
}

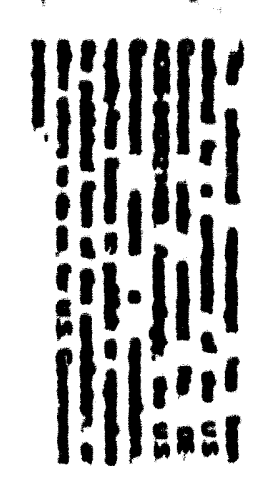

\section{AISTILACT}

Production of eingly and doubly charged lielium lons by impact of keV photons is etudied. The ratio $R_{p h}=\sigma_{p h}^{++} / \sigma_{p h}^{+}$for photoabsorption is calculated in the photon. enorgy range 2-18 keV using correlated initial. and final-state wave functions. Extrap. olation towards nsymptotic photon nnargies yiolds $R_{p h}(\omega \rightarrow \infty)=1.60 \%$ in agreement with previous predictions. Ionization due to Compton scattering, which bncomes com. parable to photoabsorption above $\omega \sim 3 \mathrm{keV}$, is discussed.

\section{INTRODUCTION}

Many-electron transitions in atomic systems inducod by photon impact are of considerable Interest since the Hamiltonian coupling of the electronic degrees of freedom to the electromagnetic field is bullt up of one-body operntors. A transition involving more than one electron must therefore procced via the interelectronic interaction (correlation). The simplest systems for studies of these processes ara two-electron atoms and lons. Considerable work was carried out in the late 50s and early 60 s on the dipole matrix elements for two-electron transitions in helium for the purpose of evaluating the Lamb shift of the ground state. ${ }^{1-3}$ In the late $60 \mathrm{~s}$, when measurements of the ratio $R_{p h}=\sigma_{p h}^{++} / \sigma_{p h}^{+}$of the double- to single-photoionization cross sections were reporled from threshold up to $625 \mathrm{eV}, 4$ it was realized that this quantity is very sensitive to the usage of highly accurate wave functions. ${ }^{3-10}$ Apart from the theoretical efforts to obtain $R_{p h}$ for photoabsorption in the low-energy regime, predictions of the non-relativistic asymptotic value $R_{p h}(\omega \rightarrow \infty)$ also became available. ${ }^{5-8}$ The experimental verification of this fundamental quantity has become possible only very recently with the advent of synchrotron-light sources having sufficient intensity. This progress on the experimental side $^{11-13}$ has stimulated renewed theoretical interest ${ }^{14-19}$ in double ionization of He at high photon energies.

A complication in the interpretation of the experiments arises, however, when the photon energy exceeds approximately $3 \mathrm{keV},{ }^{20}$ The photolonization cross section decays rapidly as $\omega^{-7 / 2}$ while the Compton scattering cross section is essentially independent of $\omega$ in this energy regime. The cross sections are equal at about $6 \mathrm{keV} .{ }^{2 i}$ Based on the energy transfer to the atomic system, the approximate thresholds for single- and doubleionization due to inelastic Compton scattering are 2.5 and $4.5 \mathrm{keV}$, respectively. Since the present experiments cannot distinguish between these two competing processes, the measured ratio $R$ is expected to be a weighted average of $R_{p h}$ and the corresponding ratio for Compton scattering $R_{C}$. Above $\sim 8 \mathrm{keV}$ the experimentally measured $R$ is exclusively determined by Compton scattering $\left(R=R_{C}\right)$.

- Present address: Manne Siegbahn Institute of Physics, S-10105 Stockholm, Sweden 
We present calculations of lonization-cxcitation and double lonization crosesections for photoabsorption in the 2-18 keV energy range einploying correlated initial and final states and sum rules. We discuss the single and double ionization process by Compton scattering and catimate the contribution to the apparent $R$ as measured by the recent experiments.

\section{TIIEONY}

The crose section for lonization of one electron Into a continuum state labeled by the momontum $k$ and angular momentum quanlum numbers $l$ and $M$ nnd simultaneous excitation of the other electron to a $110^{+}(\mathrm{n} / \mathrm{m})$ state by photoabsorption is, in the dipolo approximation, given hy (we use atomic units throughout unluss otherwiso stated)

$$
\sigma_{p h}^{+*}(k L M, n l m)=\frac{2 \pi^{2}}{c} \int d E \frac{d f(k L M, n l m)}{d E} \delta\left(E+E_{n}-\omega+l_{1}\right) .
$$

where $c$ is the speed of light, $d(k L M, n / m) / d E$ is the oscillator strength for the transition from ground state helium to a bound $\mathrm{Ho}^{+}(\mathrm{nlm})$ stato and a continuum state $(k L M)$ with energy $E=k^{2} / 2, w$ is the incident photon energy, $I_{1}$ is the first lonization potential of Ile, and $E_{n}$ is the excitation energy of the $n$-mnnifold of Ilet measured from the ground statu.

The acceleration gauge of the oscillator strength is

$$
\frac{d f^{A}(k L M, n l m)}{d E}=\frac{2 k}{\omega^{3}}\left|\left\langle k \cdot L M, n l m\left|\left(\nabla_{1} V+\nabla_{2} V\right)_{1}\right| i\right\rangle\right|^{2},
$$

where $V$ is the atomic potential energy and tho polarization direction is taken along the $x$ axis. Alternatively, the oscillator strength can be expressed in the length and velocity forms. With exact initial- and final-state wave functions the various gauges of the oscillator strengths are equivalent while for approximate wave functions this is, in general, not true. The sensitivity of the oscillator strength to the gauge provides in the latter case a measure of the quality of the wave functions.

For the ground-state of He we use a 20-parameter Hylleraas-type wave function 22 and for the final state we use a wave function of the form

$$
\Psi_{\mathrm{k}, n / m}^{(-)}\left(\mathbf{r}_{1}, \mathbf{r}_{2}\right)=\frac{1}{\sqrt{2}}\left[\Phi_{n l m}\left(\mathbf{r}_{1}\right) \Phi_{\mathrm{k}}^{(-)}\left(\mathbf{r}_{2}\right) D_{\mathbf{k}_{12}}^{(-)}\left(\mathbf{r}_{12}\right)+\mathbf{r}_{1} \leftrightarrow \mathbf{r}_{2}\right]
$$

where $\Phi_{n / m}$ and $\Phi_{k}^{(-)}$are bound and continuum wave functions defined in the unscreened field of the $\mathrm{He}^{2+}$ nucleus and

$$
D_{\mathrm{k}_{12}}^{(-)}\left(\mathrm{r}_{12}\right)=\exp (-\pi \alpha / 2) \Gamma(1-i \alpha)_{1} F_{1}\left[i \alpha, 1,-i\left(k_{12} r_{12}+k_{12} \cdot \mathrm{r}_{12}\right)\right]
$$

is a Coulomb distortion factor which accounts for the elcctron-electron interaction. The continuum states $\Phi_{k}^{(-)}$are normalized to a $\delta$ function on the momentum scale. In (4) $\mathrm{k}_{12}=\mathrm{k} / 2$ is the interelectronic momentum and $\alpha=1 /\left(2 k_{12}\right)$. The states $\left|k_{i} L M, n l m\right\rangle$ in (2) are obtained by expanding $\Phi_{k}^{(-)}$and $D_{k_{12}}^{(-)}$in partial waves and recoupling to ( $L M$ ) states. 
In ofder to obtain the cotal croses section for lonizing one electron and leaving the second electron bound to the nucleus, the lonlzation excitasion cross sectlons (1) are summed over all bound states and over angular momenta of the continuum electron

$$
\sigma_{p h}^{+}(w)=\sum_{l, N} \sum_{n \mid m} \sigma_{p h}^{+\bullet}(k L M, n l m)
$$

with $L=1+1$ and $M+m=0$.

The cross section for double lonization with ejection of two electrons having energies $E=k^{2} / 2$ and $E^{\prime}=k^{\prime 2} / 2$ can be defined in analogy to (1) at

$$
\frac{d \sigma_{p h}^{++}\left(k L M, k^{\prime} I m\right)}{d E^{\prime}}=\frac{2 \pi^{2}}{c} \int d E \frac{l^{2} f\left(k L M, k^{\prime} I m\right)}{d E^{\prime} d E^{\prime}} \delta\left(E+E^{\prime}-\omega+I_{2}\right),
$$

where $I_{2}$ is the total ionization potential of Ile. The total double-ionization cross section is

$$
\sigma_{p h}^{++}(w)=\sum_{L M} \sum_{l m} \int_{0}^{w-1 / 2} d E^{\prime} \frac{d o_{p h}^{++}\left(k L M, k^{\prime} / m\right)}{d E^{\prime}}
$$

In the acceleration form the oscillator strength in $(0)$ is

$$
\frac{d^{2} f^{A}\left(k L M, k^{\prime} / m\right)}{d E d E^{\prime}}=\frac{2 k k^{\prime}}{\omega^{3}}\left|\left\langle k L M, k^{\prime} \ln \left|\left(\nabla_{1} V+\nabla_{2} V\right)_{2}\right| i\right\rangle\right|^{2},
$$

and the length and velocity forms are similarly defined.

The state $\left.\left|k L M, k^{\prime}\right| m\right\rangle$ in $(8)$ is the analogue to (3) obtained by replacing the bound state $\Phi_{n / m}$ by $\Phi_{k^{\prime}}^{(-)}$, by partial wave expanding the two continuum wave functions and the distortion factor, and by recoupling to angular momenta $(L M)$ and $(1 \mathrm{~m})$ of each electron.

In the case of two continuum electrons the Sommerfeld parameter $\alpha$ in $D^{(-)}$depends on the relative angle between the emission directions of the two electrons. This complicates the direct evaluation of $\sigma_{p h}^{++}$as compared to ionization-excitation cross sections. However, the problem can be circumvented by employing a closure approximation. At high energies the photo electron carries nearly all the available energy $\omega-I_{2} \approx \omega$, and the second electron is 'shaken up' to a low-lying continuum state. ${ }^{17,10}$ The error introduced by fixing the energy of the fast electron and extending the upper limit in the integration in (7) to infinity is therefore small at higl: photon energies. The sum of single- and double-ionizalion cross sections

$$
\sigma_{S}=\sigma_{p h}^{+}+\sigma_{p h}^{++}=\sum_{L M} \sum_{l m}\left[\sum_{n} \sigma_{p h}^{+*}(k L M, n l m)+\int_{0}^{\omega-l_{2}} d E^{\prime} \frac{d \sigma_{p h}^{++}\left(k L M, k^{\prime} l m\right)}{d E^{\prime}}\right]
$$

can be evaluated with these approximations by using the closure property of the $\mathrm{He}^{+}$ eigenfunctions. The double ionization cross section can now be obtained without reference to the two-electron continuum states, as $\sigma^{++}=\sigma_{S}-\sigma^{+}$In the limit $\omega \rightarrow \infty$ this procedure becomes exact. ${ }^{2,23}$

Theoretical investigations of Compton scattering of bound electrons are usually re. stricted to the coherent and incoherent cross sections. ${ }^{24}$ For the problem at hand, this approach cannot be applied since we are concerned with final-state specific processes. 
A high-energy appronch a deacribed above for photosbarpticn is not justified because the dominant onergy transfers $\Delta E$ from the photon to the electron(s) rangen from zero to an upper limit $\Delta E_{\text {mee }}$ approximately given by the value for Compton scaltering off free electrons

$$
\Delta E_{\text {max }}=\frac{2 \omega^{2}}{m c^{2}+2 \omega}
$$

and the distribution of energy transfers is esentially independent of $\Delta E$ in this range. One othar important distinction between Compton scattering and pliotoabsorption is the distribution of angular moinenta in the final state. While for photonbsorption only the final-state $P$ sector is reached from the ground state of 110 (in the dipole approximation), a large number of Anal-state angular momenta will contribute to the transition amplitude for Compton scattering.

In order to estimate the influence of Compton scattering on the measurad $R$ we make here an impulse (or 'binary-encounter') approximation to obtain the Compton cross section differential in the energy transfer $\Delta E$

$$
\frac{d \sigma_{C}(\omega)}{d \Delta E}=\int d^{3} q \int_{0}^{p_{\text {mac }}} d p \frac{p}{c} \delta\left(q \cdot p+p^{2} / 2+c_{B}-\Delta E\right)|\phi(q)|^{2} \frac{d \sigma_{\kappa N}(\omega)}{d p}
$$

where $p_{m a s}$ is the electronic momentum corresponding to maxinum energy transfer (see Eq. 10) in a binary encounter between the photon and one olectron, $\epsilon_{B}$ is the orbital binding energy of one electron, $\phi(q)$ is the momentum-epace wave function of the ono electron in the ground state, and $d \sigma_{K N}\left(\omega^{j}\right) / d p$ is the free-electron Compton cross section differential in the momentum transfer to the electron for which we use the Klein-Nishina formula. The single-ionization Compton cross section $\sigma_{C}^{+}(u)$ is then obtained by integrating (11) between $I_{1}$ and $\omega$ and multiplying by two to account for the number of electrons. Fur double ionization we use

$$
\sigma_{C}^{++}(\omega)=2 \int_{l_{2}}^{\omega} d \Delta E \frac{d \sigma_{C}(\omega)}{d \Delta E} R_{C}(\Delta E)
$$

where $R_{C}(\Delta E)$ specifies the ratio of double to single ionization at a given energy transfer. Of course, the exact knowledge of $R_{C}(\Delta E)$ would imply that the problem at hand was solved. We make here the following approximation: for final states in the $P$ sector $R_{C}^{L=1}(\Delta E)$ is assumnd to equal the photoabsorption ratio at the photon energy $\Delta E$, for higher angular momenta in the final state the shake-off value $R_{C}^{i>1}(\Delta E)=0.73 \%$ is used. The justification for this approximation relies on calculations for jonization. excitation by Compton scattering ${ }^{24}$ described below.

\section{RESULTS AND DISCUSSION}

While it has been established that the ionization-excitation and double iunization cross sections are independent of correlation in the final state as $\omega \rightarrow \infty$, provided an accurate wave function of the initial state is used, $3,6,25$ this is not the case at finite $\omega$. We illustrate this for $\omega=2 \mathrm{keV}$ in Fig. 1 where the difference $\Delta B=B_{\text {corr }}-$ $E_{\text {uncorr }}^{\prime}$ of the branching ratios $B(n)=\sigma_{p h}^{+*} / \sigma_{S}$ calculated with and without the finalstate distortion $D^{!-1}$ are shown. The effect of fillal-state correlation is to redistribute probability for iunization without excitation to the ionization-excitation and doubleionization chamuels. 


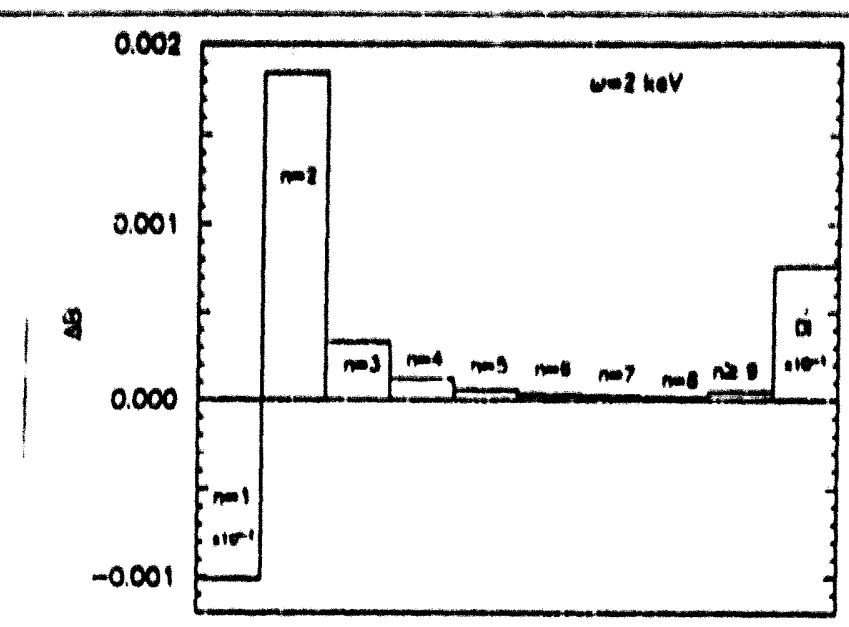

Figure 1: The change $\Delta B$ in the branching ratios $B(n)=\sigma_{p h}^{+*}(n) /\left(\sigma_{p h}^{+}+\sigma_{p h}^{++}\right)$calculated with and willout correlation in the final-stato wave function. The bars for $n=1$ and duable ionization (DI) have been multiplied by $10^{-1}$.

In Fig. 2 we show our rexult for $R_{p h}$ as function of $\omega^{-1}$ together with the recent calculations by Teng and Shakeshaft, ${ }^{17}$ Hino, ${ }^{18}$ and the MBPT calculation by Hino et al. ${ }^{10}$ In the calculations of Hino and of Teng and Shakeshaft the double-ionization cross section was calculated directly using the two-electron continuum analogue to the final state (3). Teng and Shakeshaft used the velocity form of the dipole operator, while Hino used the acceleration form but took only the monopole contribution from the distortion factor $D^{(-)}$into account. The MBPT calculation used various forms of the dipole operator. The acceleration form, shown in Fig. 2, the length and velocity forms all give similar results in the high-energy region. ${ }^{10}$

Our present result reaches an $\omega^{-1}$ behavior for $\omega>5 \mathrm{keV}$ and extrapolation to infinite photon energy yields the correct non-relativistic limit for photoabsorption $R_{p h}(\infty)=1.06 \%$, which was obtained much earlier by other authors ${ }^{5,8}$ using only a correlated initial state. The value of the coefficient of the leading $\omega^{-1}$ term is $0.90 \mathrm{keV}$. The short-dashed line in Fig. 2 represents an extrapolation of this linear behavior in $\omega^{-1}$ to both larger and smaller energies.

The various calculations ${ }^{17-19}$ do not converge to the correct high-energy limit, even though they differ by relatively small amounts. We attribute this discrepancy to inaccurate initial-state wave functions used in the calculations. For $\omega^{-1}>0.2$ the results start to diverge significantly. It is important to realize that the final state (3) and its two-electron continuum analogue constitute high-energy approximations and their use is not justified for lower photon encrgies. Our present result and the result of Teng and Shakeshaft have very similar slopes from 8 down to about $4 \mathrm{keV}$. At lower $\omega$ our result has a much stronger dependence on $\omega^{-1}$. We have traced this strong dependence to the contributions from the $l \neq 0$ multipoles of $D^{(-)}$. A part from the validity of the final state (3), the accuracy of the present result relies on the accuracy of the closure approximation. This approximation breaks down if the energy sharing between the two electrons is not highly asymmetric. We have verified for an uncorrelated final state that the accuracy is sufficient at least down to $\omega=2 \mathrm{keV}$. Ilowever, the validity of the 


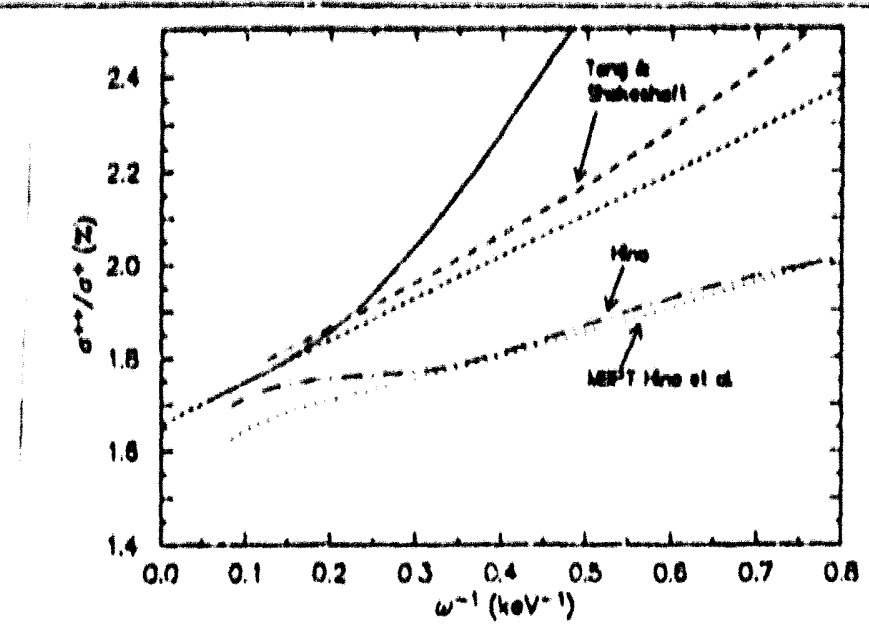

Figure 2: The ratio $R_{p h}=\sigma_{p h}^{++} / \sigma_{p h}^{+}$for pholonbsorption as functicn of $\omega^{-1}$. Solid curve: present result; long-dashed curve, Teng and Slinkeshafh;" dash-dotled curve, Ilino; ${ }^{18}$ dotled curve, Hino et al. ${ }^{10}$ Short-dashed curve: the high-energy behavior of the present result accurate to order $\omega^{-1}$.

closure method for correlated final states at low energies remains to be verified.

In Fig. 3 is illustrated the excitation-ionization by inelastic Compton scaltaring. We show the ratios belween the cross sections for ionization and excitation to $\mathrm{He}^{+}(5 s)$ and Ionization without excitation as functions of the energy $E$ of the ionized electron, broken down into the final-state angular-momentum components. Wo find the angular. momentum decomposition, shown here at $\omega=10 \mathrm{keV}$, to be approximately universal functions of the energy transfer but only weakly dependent on the primary photon energy. The two arrows indicate the asymptotic ratios for photonbsorption and for shake-off, 15 respectively. As can be seen, the $L=1$ curve is close to the former value over a significant range of $E$, while the average of the $L>1$ ratios, weighted by their partial cross sections, is close to the latter value. (The $L=0$ component is small except for the lowest $E$ and does not significantly contribute to the total cross section.) It is this observalion which motivates our choice for $R_{C}^{L}(\Delta E)$ discussed in the context of Eq. (12).

In Fig. 4 is shown the ratio $R_{p h}$ for photoabsorption (dash-dotted curve) together with the corresponding ratio for Compton scattering $R_{C}$ (dotted curve) and the weighted mean of both processes (solid curve) which should be compared to the experimental points. The agreement is, considering the simplicity of the approximation, satisfactory. We also show the linear extrapolation of the photoabsorption ratio (dashed curve) which appears to improve the agreement with the experiments below $\omega=3 \mathrm{keV}$. Further work on two-electron processes by photoabsorption and inelastic scattering of photons is in progress.

\section{ACKNOWLEDGEMENTS}

This work was supported in part by NSF; by U.S. DOE, Office of Basic Energy Sciences under contract No. DE- $\Lambda$ C05-810R21400 with Martin Marjetta Energy Systems Inc.; and the Swedish Natural Science Research Council (NFR). 


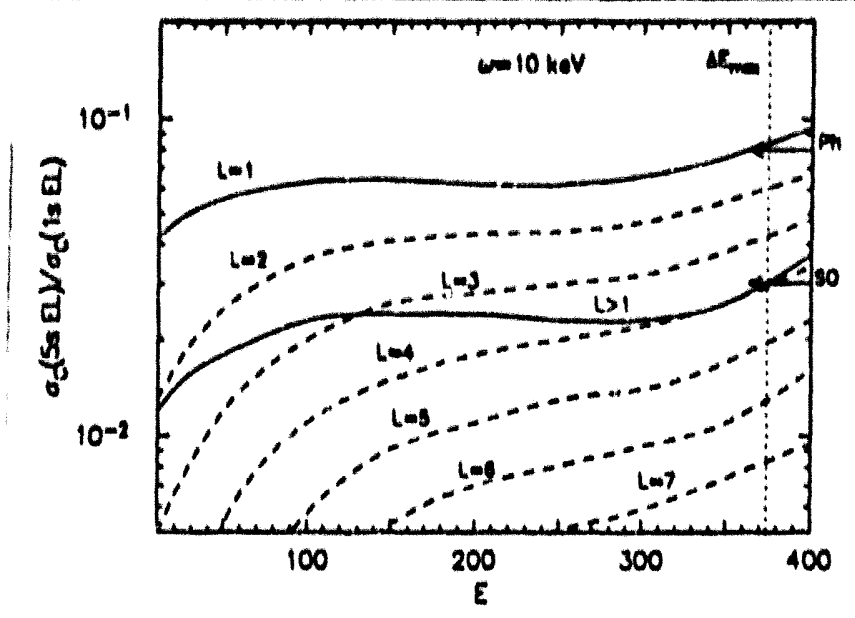

Figure 3: Ratios between the cross sections for ionization and excitation to 5s and ioniza. tion without excilation as functions of the energy of the ejected electron. The dashed curve represents the ratio for all final-states with $L>1$. Arrows indicate the asymptotic ratios for photoabsorption and shake-off.

\section{REFERENCES}

1 P.K. Kabir and E.E. Salpeter, Phys. Rev, 108 (1957) 1256.

2 A. Dalgarno and A.L. Stewart, Proc. Phys. Soc. London 76 (1960) 49.

3 E.E. Salpeter and M.H. Zajdl, Phys. Rev. 125 (1962) 248.

4 T. Carlson, Phys. Rev, 158 (1967) 142.

F.W. Byron Jr, and C.J. Joachain, Phys. Rov. 164 (1967) 1.

T. Aberg, Phys. Rev. A 2 (1970) 1726.

7 R.L. Brown and R.J. Gould, Phys. Rev. D 1 (1970) 2252.

8 M. Ya. Amusia, E.G. Drukarev, V.G. Gorshkov, and M.P. Kazachkov, J. Phys. B: At. Mol. Pliys. 8 (1975) 1248.

R.L. Brown, Phys. Rev. A 1 (1970) 341.

10 R.L. Brown, Phys. Rev. A 1 (1970) 586.

11 J.C. Ievin, D.W. Lindle, N. Keller, R.D. Miller, Y. Azuma, N. Berrah Mansour, H.G. Berry, and I.A. Sellin, Phys. Rev. Lett. 67 (1991) 968.

12 J.C. Levin, I.A. Sellin, B.M. Johnson, D.W. Lindle, R.D. Miller, N. Berrah Mansour, Y. Azuma, H.G. Berry, and D.-H. Lee, Phys. Rev. A 47 (1993) R16.

13 R.J. Bartlett and J.A.R. Samson, (private communication) (1993).

14 T. Ishihara, K. Hino, and J.H. McGuire, Phys. Rev. A 44 (1991) R6980.

15 A. Dalgarno and H.R. Sadeghpour, Phys. Rev. A 46 (1992) R3591.

16 L.R. Andersson and J. Burgdörfer, Phys. Rev. Lett., in press (1993).

17 Z. Teng and R. Shakeshaft, Phys, Rev. A 47 (1993) R3487.

18 K. Hino, Phys. Rev. A 47 in press (1993).

19 K. Hino, T. Ishihara, F. Shimizu, N. Toshima, and J.H. McGuire, Phys. Rev. A, in press (1993).

20 J.A.R. Samson, C.H. Grecne, and R.J. Bartlett, Phys. Rev. Lett., in press (1993).

21 D.E. Cullen, M.H. Chen, J.H. II ubbell, S.T. Perkins, E.F. Plechaty, J.A. Rathkopf, and J.H. Scofield, UCRL-504000, Vol. 6 Part A, Rev, 4 (Lawrence Livermore Na- 


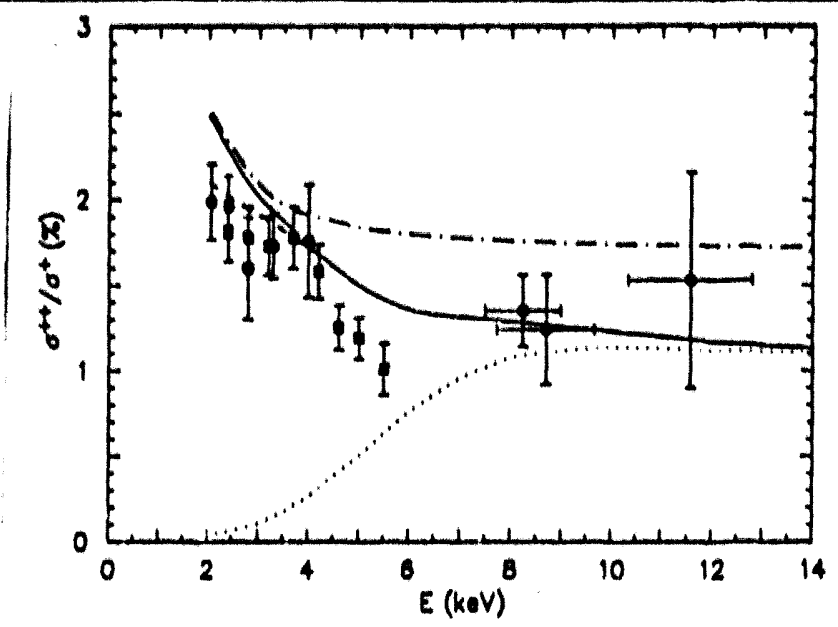

Figure 4: The ratio of double to single ionization of He by photon impact. Dash-dotted curve, photoabsorption; dotted curve, Compton scattering; solid curve, weighted mean of both processes; Dashed curve, linear extrapolation of the high-energy behavior of $R_{p h}$ to order $\omega^{-1}$, corrected for Compton scattering. Experimental data: open circle, Levin et al. ${ }^{11}$ solid circles, Levin et al:; ${ }^{12}$ solid squares, Bartlett and Samson. ${ }^{13}$

tional Laboratory) (1989).

22 J.F. Hart and G. Herzberg, Phys. Rev. 106 (1957) 79.

23 A. Dalgarno and R.W. Ewart, Proc. Phys. Soc. London 80 (1962) 616.

24 J.H. Hubbell, Wm.J. Veigele, E.A. Briggs, R.T. Brown, D.T. Cromer, and R.J. Howerton, J. Phys. Chem. Ref. Data, Vol. 4, No. 3 (1975) 471.

23 L.R. Andersson and J. Burgdörfer, (to be published)

\section{DISCLAIMER}

This report was prepared as an account of work sponsored by an agency of the United States Government. Neither the United States Government nor any agency thereof, nor any of their employees, makes any warranty, express or implied, or assumes any legal liability or responaibility for the accuracy, completeness, or usefulness of any information, apparatus, product, or process disclosed, or represents that its use would not infringe privately owned rights. Reference herein to any specific commercial product, process, or service by trade name, trademark, manufacturer, or otherwise does not necessarily constitute or imply its endorsement, recommendation, or favoring by the United States Government or any agency thereof. The views and opinions of authors expressed herein do not necessarily state or reflect those of the United States Government or any agency thereof. 


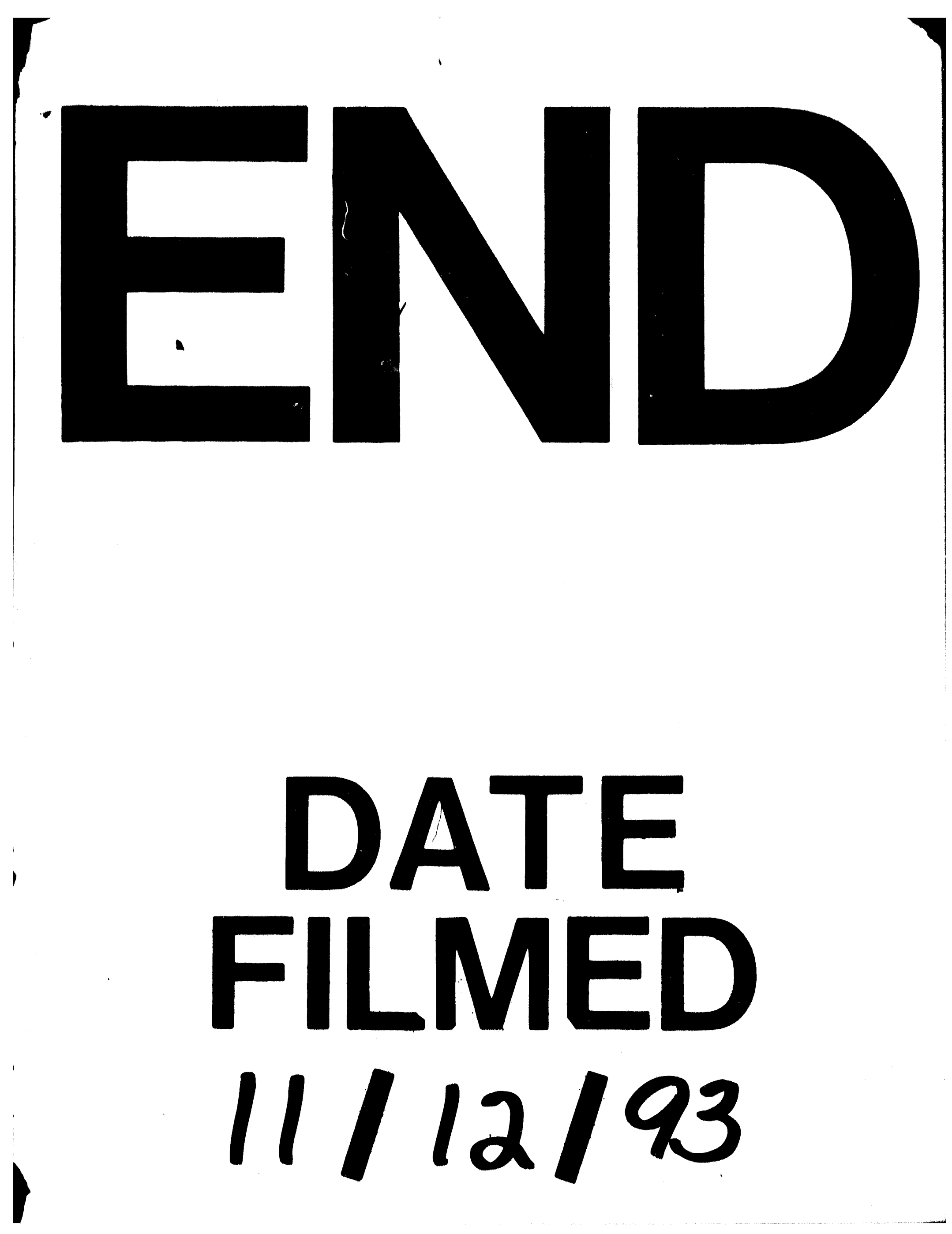




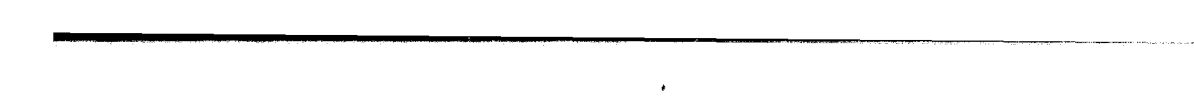

s. 